\title{
La violencia de la publicidad contra la mujer ${ }^{1}$
}

María Gómez y Patiño² | mariagp@unizar.es

UNIVERSIDAD DE ZARAGOZA

Resumen:. La cultura oficial masculina no ha sabido establecer una representación femenina adecuada y en el siglo XXI se siguen perpetuando los estereotipos sobre la mujer especialimente en la publicidad mediática. A pesar de las teorías feministas en torno a la imagen de la mujer, no se han encontrado vías para erradicar la violencia simbólica que genera un efecto que permea oda la sociedad. En este trabajo se presenta y analiza un caso ilustrativo, cuyo tratamiento publicitario (verbal e icónico) no favorece la igualdad, mientras que incrementa la discriminación y el sexismo.

Palabras clave: Violencia simbólica; publicidad; estereotipos; discriminación; feminismo.

Abstract: The official male culture has failed to establish an adequate representation of women in the $21^{\text {st }}$. century where traditional women's stereotypes are being perpetuated specially in media advertising. Despite most feminist theories on women's image, no solution to solve the problems has been found to erradicate the symbolic violence which generate a permeability effect on society. This paper presents and analyzes a case study, whose advertising treatment (iconic and verbal) that does not favour equality, but increases discrimination and sexism.

Key words: Symbolic violence, advertising, stereotypes, discrimination, feminism.

1 Trabajo realizado bajo el auspicio del S115 - GICID (Grupo de Investigación en Comunicación e Investigación Digital) BOA 195, de fecha 05/10/2012. RESOLUCIÓN de 26 de septiembre de 2012.: pp: 20788-804.

2 Doctora UCM (1996). PDI (1991). Imparte Univ. Zaragoza: Periodismo. Libros: Paz: Femenino, singular (2005), Las Huellas de la violencia invisible (2005), Los intangibles del turismo (2012). Traducciones: El proceso de Opinión Pública (2000), y Journals. Fundadora Seminario Permanente Estudios sobre la Mujer. Madrid. G.I. GICID. 


\section{Introducción}

En los albores del siglo XXI, se hace necesario depurar y descartar los estereotipos patriarcales en torno a la mujer que han sido creados histórica y tradicionalmente por la cultura oficial masculina, que no ha sabido entender las representaciones femeninas socialmente aceptadas por las mujeres, produciendo una gran brecha entre lo que los hombres consideran lo femenino y lo que las mujeres entienden por este mismo concepto.

Las representaciones de la mujer han sido larga y profusamente estudiadas por muchas teóricas españolas, entre otras, (Aguado et al, 1994; Almeida \& Gómez y Patiño, 2005; Amorós, 1994, 1996; Bernárdez Rodal, 2010; Camps, 1990; Durán, 2000; Radl Philipp, 1996; Valcárcel, 2003), europeas (Arendt, 1995; Buzzatti \& Salvo, 2001; Nash, 1994; Scott, 1990); y americanas (Deaux, 1985; Eagly \& Johnson,1990; Chiapello \& Fairclough, 2002; Hare-Mustin \& Marecek, 1988; Offen, Roach Pierson \& Rendall, 1991; Reay \& Ball, 2000; Sendercock \& Forsyth, 1992; Wollstonecraft, 1998). Sin embargo, existe un gran vacío en la cultura tradicional masculina, que sigue sin resolverse, según ilustra el caso que se presenta, pero que paradójicamente ya fue diagnosticado por Concepción Arenal en $1895^{3}$, en un artículo titulado "Estado actual de la mujer en España" publicado en el Boletín de la Institución Libre de Enseñanza el 31 de agosto, cuya explicación, referida a los hombres, es tan clara como contundente:

\footnotetext{
"aún siendo ilustrados en ciertos asuntos, son muy ignorantes en éste, y tienen inclinaciones de sultán, reminiscencias de salvaje y pretensiones de sacerdote, queriendo ser escuchados como oráculos, obedecidos como señores, y amados con una fidelidad a que no se creen en el caso de corresponder (...). Aunque acicalen su cuerpo y cultiven algo su espíritu, (...), no ven todavía en la mujer más que la hembra, (...). Por extraño que parezca, es el caso de la mayoría de los hombres, aun de los que han recibido alguna instrucción".
}

A Concepción Arenal le producía tanta extrañeza esta constatación como producen discursos en el siglo XXI, como el analizado. El caso estudiado permite revisar todas las dimensiones analíticas que Joan W. Scott (1990) proponía: 1) simbólica, 2) normativa, 3) sistémica y 4) identidad subjetiva. Desde la primera dimensión: la violencia simbólica permite comprender los fenómenos entendidos como violentos, que se siguen produciendo reproduciendo en todos los medios de comunicación social y en todas sus vertientes: radio, cine, televisión, prensa escrita, internet, y publicidad, y ésta a su vez en todos los soportes posibles. Desde la segunda en inglés diez años antes por Mr. Stanton (New York, Putnam's Sons, 1884). 
dimensión, se pueden cuestionar las normas sociales, políticas y legales. Desde la tercera dimensión, se debe problematizar sobre la función de las instituciones y organizaciones sociales, como la estudiada en este caso, y como cuarta dimensión sobre la identidad genérica femenina que se está perpetuando sin solución de continuidad.

Si bien para la mayor parte de los mass media la audiencia ha de ser activa y su exposición a los medios selectiva (Böckelmann, 1975; Festinger, 1957; Klapper, 1974), en el fenómeno publicitario, los consumidores son pasivos, pese a su voluntad, lo que genera una permeabilidad que va más allá de sus deseos, produciendo un efecto cuya onda de reproducción penetra en toda la sociedad casi por igual, perpetuando o magnificando este tipo de mensajes cargados de violencia simbólica, que pasan inadvertidos para los mismos creadores, que no parecen identificarlos fácilmente. Siendo así, han de ser los diferentes colectivos feministas, analistas críticos y atentos, los que han de generar la alarma ante la aparición y reproducción de estos estereotipos. En ocasiones pueden ser intangibles, y poco visibles, pero más que la conveniencia, aparece la necesidad de eliminarlos. En este trabajo se analiza un caso, producido el verano 2013 con el anuncio del Sorteo Extraordinario del Turista, Lotería Nacional (Imagen 1), que tuvo que ser retirado por machista, lo que ejemplifica cómo la dominación patriarcal sigue actuando, reproduciendo estos estereotipos creados, perpetuados y fomentados por la cultura patriarcal dominante. Este tratamiento publicitario (verbal e icónico) es más frecuente de lo deseable y su eliminación totalmente necesaria. Esta violencia simbólica contra las mujeres, favorece la desigualdad de géneros, la discriminación y el sexismo, por lo que su minimización, ruptura o eliminación son cada vez más urgentes.

Imagen 1: Anuncio estudio-caso

\section{El impacto de la autoprofecía que se cumple}

Hablar de violencia simbólica en el siglo XXI remite necesariamente a siglos anteriores $y$ especialmente al siglo $X X$, periodo de tiempo en el que se han construido la mayor parte de las teorías al respecto, e incluso al XIX, como se indicaba en el epígrafe anterior. No se pretende presentar una revisión de todo lo establecido hasta el momento, e inicialmente el concepto de violencia simbólica sirve como una categoría explicativa de los fenómenos entendidos como simbólicamente violentos, que se siguen reproduciendo bajo el paraguas de los medios de comunicación social en todos sus géneros: radio, cine, televisión, prensa 
escrita e internet. En todos ellos está presente la publicidad, aspirando a conseguir los mensajes más persuasivos.

El siglo XX fue muy productivo en cuanto a la aparición de las teorías explicativas de las relaciones de dominación, y más concretamente en cuanto a la dominación masculina sobre la femenina, no exenta de fuerza, violencia y poder. Como base para la construcción de estas teorías explicativas, se tomaban las teorías clásicas de Friedrich Marx, Emile Durkheim y Max Weber, que permitía no reiniciar un camino ya andado sobre la fundamentación del poder. Más recientemente Pierre Bourdieu ha retomado el tema con toda la profundidad posible, teniendo en cuenta la diferencia de niveles asignados a cada uno de los sexos en la dinámica de los intercambios simbólicos. Ha de recordarse que tradicionalmente los hombres han sido considerados los poseedores del capital simbólico, y que una parte de ese capital estaba constituido por un "objeto humano": la mujer.

Para sintetizar la situación, el cambio o intercambio de roles no está resultando una tarea ni fácil ni rápida, a juzgar por los casos como el presentado en esta ocasión. En opinión de Bourdieu (2000:140-141) requerirá de: "una acción colectiva que busque romper prácticamente el acuerdo inmediato de las estructuras incorporadas y de las estructuras objetivas, es decir de una revolución simbólica capaz de poner en cuestión los fundamentos de la producción y de la reproducción del capital simbólico, y en particular de la dialéctica de la pretensión y de la distinción que se halla en la raíz de la producción y del consumo de bienes culturales como signos de distinción".

Como afirma Ávila-Fuenmayor (2007), el poder es una estrategia. El caso aquí presentado es una muestra más, aunque inexplicable, de esta lucha simbólica, inconsciente quiero creer, entre los dos sexos: el dominante y el dominado.

En último extremo, no se puede ignorar lo que afirmó categóricamente Foucault (2000:28): el poder es esencialmente lo que reprime". Siendo así, se entiende perfectamente la afirmación de Ávila-Fuenmayor (2007) referido a Foucault, quien ponía su mirada en las distintas formas de dominio del hombre sobre el hombre, en este caso, de la mujer, añadiendo que verdad, saber y poder están íntimamente relacionados.

Lo que aquí se plantea es el análisis del lenguaje masculino, verbalizado a través de un anuncio publicitario de una institución de ámbito estatal, que sorprende e indigna a la comunidad de mujeres, reaccionando en contra hasta la retirada del anuncio en 
cuestión. Aparentemente estas instancias que aspiran al ejercicio de un poder de imposición simbólica se sumergen innecesariamente en unas relaciones de competencia y de fuerza que ponen de manifiesto la tensión existente y que al parecer no está resuelta (Fernández, 2005).

Estas relaciones de competencia, o de fuerza simbólicas, parecen tener una legitimación indivisible. Para Bourdieu y Passseron (2002:34): "las reivindicaciones de legitimidad hallan su fuerza relativa, en último término, en la fuerza de los grupos o clases de las que expresan, directa o indirectamente, los intereses materiales y simbólicos". Es precisamente en este punto en el que los intereses materiales y simbólicos masculinos y femeninos se enfrentan con una violencia simbólica totalmente visible.

En La dominación masculina, ensayo sobre las diferencias entre los sexos, Bourdieu (2000:11-12) ya apuntaba que la dominación masculina y la forma en que ha sido impuesta y tolerada, constituyen el mejor ejemplo de sumisión, y que ha dado lugar a lo que él tan bien ha definido como "violencia simbólica, violencia amortiguada, insensible, e invisible para sus propias víctimas, que se ejerce esencialmente a través de los caminos puramente simbólicos de la comunicación y del conocimiento"

Es muy acertada la afirmación de Nancy Piedra Guillén (2004) cuando dice que el poder se ejerce a partir de unos dispositivos de poder, contenidos en las construcciones sociales, sin que por ello se deba victimizar a las mujeres. Esta violencia simbólica e invisible está presente en estos dispositivos de poder, que pueden adquirir multitud de formas. Este modo de violencia invisible (Gómez y Patiño, 2002) han actuado de una forma tan sutil e imperceptible que está difuminada y desdibujada, resultando incluso invisible para las propias víctimas. Las peores consecuencias son también imperceptibles, porque en la medida de la capacidad percusora de los mensajes, la fuerza de la profecía self-fulfilling prophecy. Se auto-cumplirá. Sin que se haya podido dar lugar a una reacción equilibradora y restauradora de la situación anunciada (Merton, 1948). En otras palabras, si estos mensajes se difundieran, podrían generar fácilmente el efecto de la profecía que se cumple a sí misma: la mujer se debería dejar tocar por uno de cada tres. Hecho éste que le convertiría en un objeto accesible a 1/3 de la población masculina, de lo que se desprendería la ausencia de dignidad de la mujer. Dicho lo cual, cualquier de los efectos son devastadores, por no citar el efecto perpetuación de la cultura machista dominante (Bourdieu, 2000), que una vez cumplida la profecía se perpetuaría porque el discurso de violencia simbólica dominante se seguiría perpetuando. 


\section{Profesionalidad, ética y desprecio de la ley}

En lo relativo a las dimensiones normativa y sistémica, se ha de plantear el problema desde los aspectos éticos y legales, a los propiamente institucionales. La opinión pública reclama el establecimiento de la norma (ley) para acabar con este tipo de violencias. No obstante, a pesar de la existencia de la ley, el poder económico establecido parece desatender, cuando no despreciar, la letra de la ley.

En España, a pesar de que existe una Ley General de la Comunicación Audiovisual 7/2010, de 31 de marzo, para flexibilizar los modos de gestión de los servicios públicos de comunicación audiovisual autonómicos, que en su capítulo 1, Art. 4, punto 4, dice literalmente: "La comunicación audiovisual debe respetar el honor, la intimidad y la propia imagen de las personas y garantizar los derechos de rectificación y réplica. Todo ello en los términos previstos por la normativa vigente. Diciendo asimismo, en su punto 5: "Todas las personas tienen el derecho a que la comunicación informativa se elabore de acuerdo con el deber de diligencia en la comprobación de la veracidad de la información y a que sea respetuosa con el pluralismo político, social y cultural".

Esta ley fue modificada dos años después, mediante la Ley 6/2012, de 1 de agosto, que en su art. 1, dice: Se modifican los apartados 2 y 5 del artículo 7 que pasan a tener la siguiente redacción: "2. Está prohibida la emisión de contenidos audiovisuales que puedan perjudicar seriamente el desarrollo físico, mental o moral de los menores, y en particular la de aquellos programas que incluyan escenas de pornografía, maltrato, violencia de género o violencia gratuita." A pesar de que este articulado es tan contundente como inequívoco, las tensiones de violencia simbólica se siguen produciendo en los mass media cada día, con especial incidencia en los de carácter audiovisual.

Tanto esta ley como su modificación son una ratificación precisa de la Constitución española (1978), que anticipaba estas necesidades, adelantando también la norma de obligado cumplimiento en tus artículos 10 y 18 , entre otros. Resulta incuestionable que cualquier presentación vejatoria y discriminatoria de la mujer no respeta ni los derechos democráticos en general, ni los constitucionales, en particular.

En Estados Unidos, en lo relativo a algunos temas de relaciones públicas, el código de la Sociedad Americana de Relaciones Públicas (Public Relations Society of America - PRSA) en su artículo 2, señala, "todo miembro deberá ejemplificar 
elevados estándares de honradez e integridad en cumplimiento de su doble obligación frente a su cliente y al proceso democrático." En su art. 5, indica: "Ningún miembro deberá, conscientemente, divulgar información falsa o engañosa...". El tema legal referido a la publicidad sexista ha sido ampliamente estudiado y expuesto por Navarro-Beltrá \& Martín Llaguno (2011, 2012), tanto desde la necesidad de legislar como de cumplir la ley, y lo que es menos importante, sobre la objetividad existente en su interpretación. Pero por más subjetividad que pudiera existir, hay actuaciones que no caben en la ley, ni siquiera en el sentido común. Esto conduce directamente a la dimensión sistémica que obliga a preguntar por las razones para que estos hechos se sigan produciendo en el siglo XXI con una legislación vigente, citada anteriormente. No parece aceptable la profesionalidad de las agencias publicitarias en las que la Sociedad Estatal Loterías y Apuestas del Estado (Selae) parecen confiar. Igualmente cuestionables son los responsables de comunicación corporativa de la institución, con una evidente despreocupación ética, deontológica y legal. Criterios todos ellos tan importantes que no cabe una aproximación social de las organizaciones sin una consideración ético-social (Xifra Triadú, 2010). Para una aproximación al análisis del caso, incluso habiendo sido retirado, se hace preciso preguntar una serie de puntos, que permitirán identificar el problema y evitar futuros casos: 1) por la empresa que ha realizado la campaña; 2) el responsable de comunicación corporativa que ha aceptado el anuncio, es un cargo técnico o político; 3) la sanción económica que el anuncio debería tener, dado que la retirada del cartel no parece suficiente, a pesar de que se trate, y debería pesar en contra, de una sociedad estatal, dependiente además del Ministerio de Hacienda, 4) en quién recae la responsabilidad final de la difusión de este tipo de mensajes machistas en el año 13 del siglo XXI.

\section{Algunas consideraciones en torno a la imagen y la representación femenina}

Referida a la situación de violencia simbólica, la primera consideración que se debe plantear, tanto por cronología como por importancia, es la esbozada por Concepción Arenal (1974:41): "la opinión es la verdadera causante de todas estas injusticias, porque hace la ley, o porque la infringe" . La pregunta subyacente es: ¿Para qué sirve la ley si se incumple?

Las mujeres occidentales, y más concretamente las europeas han forjado su propio modelo identitario (Gómez y Patiño, 1997) que nada tiene que ver con la manera en que las mujeres son incorporadas al discurso publicitario, dado que lo hacen a 
través de estereotipos creados por la tradición patriarcal. Estas versiones femeninas crean un modelo de mujer obediente, muda y sumisa, plegada al servicio del deseo varonil, satisfaciendo la obsesión voyerista y táctil, donde lo femenino presenta la histórica dicotomía madre/puta (María/Eva para la tradición cristiana/católica). En cambio, la identidad femenina construida por las mujeres está muy lejos de este patrón patriarcal (Gómez y Patiño, 2009, 2010), que como se observa en este caso, resulta claramente humillante para la mujer. El cuerpo femenino es solo un objeto sexual, que por el hecho de serlo constituye una degradación de la mujer, hasta su ridiculización. Se trata de un objeto sexual desprovisto de dignidad, de ética, de moral, de personalidad, y hasta de capacidad de decisión, pues la frase contiene un imperativo: la descalificación intelectual, se cuelan distintas formas de esta sutil coacción psicológica.

En ocasiones, la mujer ha tenido que mostrarse dócil y obediente para poder ejercer un poder fuertemente masculinizado (Gómez y Patiño, 2002), porque siempre ha sido consciente de lo contraproducente de lo contrario. Pero de esta docilidad $y$ obediencia estratégica no se puede inferir la misma obediencia del mandato publicitario estudiado.

Mediante la representación de una realidad plasmada por unos objetos/sujetos, se refleja y se perpetua una realidad, porque el lenguaje, tanto si es verbal, icónico o audiovisual, refracta la realidad. En ese mensaje y en su lenguaje se está transmitiendo una realidad, dado que para Bajtin (1986) la palabra es un instrumento ideológico esencial. De ahí que la realidad pueda ser representada como 1) un reflejo de la realidad existente, y 2) un proyecto de la realidad futura, como parte de una profecía que se cumplirá por ser enunciada en el escenario público (Merton, 1948).

\section{Aproximación al análisis del anuncio}

La edición digital de El País, publicaba bajo la autoría de Laura Delle Femmine el 27 de agosto, 2013 un artículo cuyo título no dejaba lugar a dudas: "Retirado un anuncio de Loterías del Estado acusado de machismo. La Fundación Mujeres denuncia que el eslogan: "Uno de cada tres quiere tocarte. Déjate" viola los derechos de la mujer ${ }^{4}$.

\footnotetext{
4 http://sociedad.elpais.com/sociedad/2013/08/27/actualidad/1377616850_626615.html (consultado el 15 de septiembre, 2013)
} 
El eslogan: Uno de cada tres quiere tocarte. Déjate, creado por Loterías y Apuestas del Estado, para promocionar el sorteo extraordinario del turista, cuya fecha de celebración era el 7 de septiembre, 2013. La polémica surgió y consiguió la retirada del anuncio. La web de la Asociación Fundación Mujeres emitió un comunicado denunciando el cartel por violación de la ley que garantiza la igualdad de género, constituyendo un "grave ataque a la dignidad de las mujeres". Según esta asociación, el mensaje "apela a comportamientos de sumisión y acoso sexual que consiguen frivolizar a la mujer" ${ }^{\prime \prime}$.

Este anuncio permite observar una clara disonancia psicológica creada por las actitudes masculina y femenina en conflicto sobre la idea general de la atracción, que hace patente la discriminación sexista socialmente determinada contra la mujer, en base a una no enunciada inferioridad. Lo cierto es que esta creencia, social, ética y legalmente sancionada, persiste en muchas culturas para justificar la discriminación y tratamiento vejatorio de la mujer. Algunos estudios explican que existen modelos de comportamiento en el liderazgo (Deaux,1985; Eagly,1990; Helgesen, 1990), siendo éste siempre masculino, ejerciendo discriminación contra la mujer. Se ha utilizado el criterio expuesto por Chiapello \& Fairclough (2002) para un análisis crítico del discurso (ACD) (Critical Discourse Análisis - CDA) y el efecto de la autoprofecía (Merton, 1948), que arrojan los siguientes resultados:

M1: Denotativo-descriptivo: Uno de cada tres quiere tocarte. Ofrece una información cuantitativa, el equivalente a $1 / 3$ de la población masculina se siente atraída por ti y quiere tocarte.

M2: Mensaje imperativo: Déjate. La orden es directa. Imperativo categórico, que no deja lugar a la opción, simplemente a la sumisión y a la obediencia.

Los valores V1, V2 y V3 que están en juego son:

V1. Mujer objeto de deseo sexual

V2. Hombres poseedores del deseo sexual que desean ejercer su derecho

V3. Mujer, sometida al dominio masculino ha de seguir las ordenes dadas en el slogan.

V3: Profecía que se cumple a sí misma: "continua siendo un objeto de satisfacción sexual: déjate"

\footnotetext{
5 http://noticias.lainformacion.com/economia-negocios-y-finanzas/publicidad/el-gobierno-califica-dedecision-acertada-que-loteria-nacional-retire-la-campana-machista_qhevRM4JMmzvbarwr4STY3/.

Consultado el 15 de septiembre, 2013
} 
Este anuncio refuerza los modelos sexistas dominantes existentes y estereotipados, que establecen un comportamiento femenino, que sólo existen en la mirada masculina, como si se tratase de un objeto animado, ordenándole simultáneamente que permita ser tocado. En esencia, se reduce a la mujer a un cuerpo, objeto del deseo tocamientos y provisto de vida y acción animado que se incluye en una erótica del poder establecido, en la que la mujer es tratada indignamente. La pregunta subyacente es la necesidad de incorporar este mensaje sexual en un anuncio de publicidad para vender décimos de lotería. Mensaje que podría ser explicado quizá por las palabras de Foucault (1991:13): "es la multiplicación de discursos sobre el sexo en el campo de ejercicio del poder mismo: incitación institucional a hablar del sexo, y cada vez más; obstinación de las instancias del poder en oír hablar del sexo y en hacerlo hablar acerca del modo de la articulación explícita y el detalle infinitamente acumulado." Mientras que es imprescindible hacer algunas referencias a lo que significa la identidad de género, la igualdad (Amorós, 1994, 1996; Durán, 2000; Radl Philipp, 1996): pueden ilustrar lo inaceptable del mensaje publicitario.

El mensaje es claramente intolerable.

Para ofrecer un ejemplo comprensivo y explicativo de la situación de flagrante discriminación, y que permite establecer un paralelismo incuestionable, se presenta otro, que atenta contra la dignidad, es racista y perpetúa el conflicto racial, con una violencia simbólica del mismo nivel:

S1: Negro: "Uno de cada tres (blancos) quiere escupirte. Déjate"

S2: Mujer: "Uno de cada tres (hombres) quiere tocarte. Déjate"

\section{Algunas conclusiones}

El estudio de caso analizado ha permitido plantear el problema desde las cuatro dimensiones (Scott, 1990) y desde todas ellas el caso es inaceptable, a lo que la violencia simbólica o los dispositivos de poder de origen foucaultiano dan una explicación.

Otra perspectiva para entender la situación, nunca para justificarla, es la definición esbozada por Merton (1980:21): referida a la ambivalencia tanto psicológica como sociológica: "(...) expectativas conflictivas con carácter normativo, definidas socialmente para un determinado cometido social, asociado con un determinado estatus (...) estas normas se expresan mediante una oscilación: pasando del 
distanciamiento a la compasión, de la disciplina a la permisividad, de un trato personal a otro impersonal". Aplicado a este caso sería deseable pensar que a título individual/psicológico, no es fácil imaginar un hombre que propugne el mandato publicitario, mientras que la ambivalencia sociológica, parecería permitirlo.

En lo relativo a la creación y comparación de estos dos slogans S1 y S2 ofrece un paralelismo tan exacto e ilustrativo que repugnaría a cualquier lector: masculino o femenino, situación que declara el slogan inaceptable e intolerable. Tras este rechazo frontal, el hecho de haber analizado este mensaje desde distintas perspectivas, permite llegar asimismo a unas conclusiones transdisciplinares, que se apoyan en algunas teorías feministas y que permiten haber realizado un análisis crítico del discurso ( $A C D$ ), que refuerzan las bases teóricas de estas disciplinas a la vez que se inicia un diálogo entre ellas.

El modelo de mensaje responde por tanto a un espíritu patriarcal dentro de una economía de mercado capitalista, en el que se sigue perpetuando y reforzando el valor (V1 y V2) de los atributos tradicionalmente atribuidos a lo "femenino" que no son otra cosa que un acto propio de la violencia simbólica dominante, donde la imagen femenina sigue siendo un objeto animado de deseo para los consumidores, supuestamente varones, o al menos un tercio de ellos, dispuestos a participar en la orden expresada en el anuncio publicitario, y que además podrían tener un tercer valor (V3) profético, según la teoría clásica mertoniana y una legitimación de este poder de dominación masculino sobre lo femenino.

Por ello, más allá del análisis específico ( $A C D$ ) quedan por resolver algunas preguntas suscitadas en la aproximación al análisis del caso, que apuntan a diferentes tipos de responsabilidades: 1) de la empresa autora de la campaña; 2) del responsable de comunicación corporativa (técnico o político); 3) la sanción económica además de la retirada del cartel, insuficiente siempre, pero más si esta institución depende del Ministerio de Hacienda, 4) de la persona encargada de la difusión de este tipo de mensajes machistas, que no son aceptables en el año 13 del siglo XXI.

Un estado democrático que, como el español, cuenta con una Constitución desde 1978, y con una legislación general a la que se suman las leyes específicas referidas a la información audiovisual no puede permitir la perpetuación de estos modelos de violencia simbólica, ya que se estaría actuando de forma perversa (sin entrar en el terreno de las intenciones) en todas las dimensiones anteriormente citadas: simbólica, normativa, sistémica y de identidad subjetiva. Son cuestiones tan serias 
para la igualdad que no pueden ser toleradas. Como afirmó Concepción Arenal en 1881: "no se puede estudiar bien el presente y el pasado sin adquirir el convencimiento de que los grandes males de los pueblos vienen menos de las injusticias que persiguen que de las que toleran" (1974:194). De ahí la importancia de las denuncias públicas, no sólo por solventar el caso concreto, sino para que estos estereotipos no se sigan reproduciendo ad infinitum.

\section{REFERENCIAS BIBLIOGRÁfICAS}

AGUAdO, Ana María. (et al.) (1994): Textos para la historia de las mujeres en España, Madrid, Cátedra.

ALMEIDA, Cristina. \& GÓMEZ y PATIÑO, María (2005): Las huellas de la violencia invisible, Barcelona, Ariel.

AMELANG, J.ames y NASH, Mary (eds.): Historia y género. Las mujeres en la Europa Moderna y Contemporánea, Valencia, Edicions Alfons el Magnànim.

AMORÓS, Celia (1994): "Igualdad e identidad", En A. Valcárcel (comp.): El concepto de igualdad, Madrid, Ed. Pablo Iglesias, pp. 29-49.

AMORÓS, Celia (1996): "Ética y feminismo", Enciclopedia Iberoamericana de Filosofía. Cuestiones morales, Madrid, Editorial Trotta/CSIC, pp. 145-71.

ARENAL, Concepción (1974): La emancipación de la mujer en España, Madrid, Júcar.

ARENDT, Hannah (1995): De la historia a la acción, Barcelona, Paidós.

ÁVILA-FUENMAYOR, Francisco (2007): "El concepto de poder en Michel Foucault", Aparte Rei. Revista de Filosofía, 53:1-16.

BAJTIN, Mijail (1986): Marxismo y filosofía del lenguaje, Madrid, Alianza.

BERNÁRDEZ RODAL, Asunción (2010): "Estrategias mediáticas de "despolitización" de las mujeres en la práctica política ( $O$ de cómo no acabar nunca con la división público/privado), CIC Cuadernos de Información y Comunicación, 15:197-218.

BÖCKELMANN, Frank (1983): Formación y funciones sociales de la opinión pública, Gustavo Gili, Barcelona.

BOURDIEU, Pierre (2000): La dominación masculina, Anagrama, Barcelona. 
BOURDIEU, Pierre \& PASSERON, J.C. (2002): La reproducción, Elementos para una teoría del sistema de enseñanza, Editorial Popular, Madrid.

BUZZATTI, Gabriella \& SALVO, Anna (2001): El cuerpo-palabra de las mujeres. Los vínculos ocultos entre el cuerpo y los afectos, Madrid, Cátedra-Instituto de la Mujer-Universidad de Valencia.

CAMPS, Victoria (1990): Virtudes públicas, Barcelona, Espasa-Calpe.

CHIAPELLO, Eve \& FAIRCLOUGH, Norma (2002): "Understanding the new management ideology: a transdisciplinary contribution from critical discourse analysis and new sociology of capitalism", Discourse Society 13:185-208.

DEAUX, Kay (1985): "Sex Differences" Annual Review of Psychology, 26:48-82.

EAGLY, Alice. H \& JOHNSON, Blair T. (1990): "Gender and Leadership Style: A Meta-Analysis" Psychological Bulletin, 108:233-56.

GEERTZ Cliffor (1990): La interpretación de las culturas, Gedisa, Barcelona.

DURÁN, María Ángeles (2000): Si Aristóteles levantara la cabeza: quince ensayos sobre las ciencias y las letras, Madrid, Cátedra.

ENDERS, V. Lorée \& RADCLIFF, Patricia B. (1998): Constructing Spanish Womanhood. Female Identity in Modern Spain, New York, Sate University of New York Press.

FERNÁNDEZ, J. Manuel (2005): "La noción de violencia simbólica en la obra de Pierre Bourdieu: una aproximación crítica", Cuadernos de Trabajo Social 18: 7-31.

FESTINGER, León (1957): A theory of cognitive dissonance, Stanford, Stanford University Press.

FOUCAULT, Michel (1991): Historia de la sexualidad, La voluntad de saber, Siglo XXI, México.

--- (2000): Defender la sociedad. Curso en el College de France (1975-1976), Fondo de Cultura Argentina, Buenos Aires.

GÓMEZ y PATIÑO, M. (1997): "Las escritoras en lengua alemana", HISTORIA Y VIDA, No Extraordinario. 1er. Trimestre. Pp. 33-41.

--- (2002): "El Nuevo mundo o el imaginario de mujer hispana" en Las Representaciones de la Mujer en la Cultura Hispánica, Valladolid, UNIVERSITAS CASTELLAE, Col. "Cultura Iberoamericana", 7:169-184 
--- (2009): "Mujeres y hombres para la diversidad. Dos componentes de un mismo constructo social", en Pilar Nova Melle/Julio del Pino Artacho (Coords.):

Sociedad y Tecnología: ¿Qué futuro nos espera?, pp. 29-36.

--- (2010): "Repensar la identidad femenina". Cuestiones de Género: de la igualdad y la diferencia, 5:541-562.

GORNICK Janet C. \& MEYERS Marcia K. (2008): "Creating gender egalitarian societies: an agenda for reform", Politics \& Society, Vol 36(3): 313-349.

HARE-MUSTIN, Rachel T. \& MARECEK, Jeanne (1988): "The meaning of difference. Gender theory, postmodernism, and psychology", American psychologist, vol. 43(6):4555-464.

KLAPPER, Joseph T. (1974): Efectos de las comunicaciones de masas, Madrid, Aguilar.

MERTON, Robert K. (1948):"The Self-Fulfilling Prophecy", The Antioch Review, $8(2): 193-210$.

--- (1980): Ambivalencia sociológica y otros ensayos, Madrid:Espasa-Calpe.

NASH, Mary (Coord.). (1994): Presencia y protagonismo. Aspectos de la historia de la mujer, Barcelona, Ediciones del Serbal.

NAVARRO-BELTRÁ, Marián \& MARTÍN LLAGUNO, Marta (2011): "El sexismo publicitario: delimitación de conceptos e indicadores de género. Estudio empírico entre estudios e indicadores de género. Estudio empírico de la producción científica", Pensar la Publicidad. Revista Internacional de Investigaciones Publicitarias, 5(1), 51-73.

http://dx.doi.org/10.5209/rev_PEPU.2011.v5.n1.3692,6263

--- (2012): "La consideración de la publicidad sexista en función del contexto y de las características personales". Pensar la Publicidad. Revista Internacional de Investigaciones Publicitarias, 6 (1), 207-230.

http://dx.doi.org/10.5209/rev_PEPU.2012.v6.n1.38663

NOVA MELLE, Pilar/Del Pino Artacho, Juan (Coords.) (2009): Sociedad y Tecnología: ¿Qué futuro nos espera?, Univ. Alcalá Henares, Madrid.

OFFEN, Karen; ROACH PIERSON, Ruth \& RENDALL, Jane (eds.) (1991): Writing Women's History. Bloomington, In: Indiana University Press.

PIEDRA GUILLÉN, Nancy (2005): "Relaciones de poder: leyendo a Foucault desde la perspectiva de género", Revista de Ciencias Sociales, 107(1):123-41. 
RADL PHILIPP, Rita María (Coord.) (1996): "Los procesos de la constitución social de las identidades de género en el contexto de la socialización humana: bases para un modelo teórico-interaccionista", en Mujeres e Institución Universitaria en Occidente. Conocimiento, investigación y roles de género. Santiago de Compostela, Universidad, pp. 15-37.

READY, Diane \& BALL, Stephen. J. (2000): "Essential of female management", Educational Management and Administration 28(2):145-159.

SANDERCOCK, Leonie \& FORSYTH Ann (1992): "A gender agenda. New directions for planning theory", Journal of the American Planning Association, $58(1): 49-59$.

SCOTT, Joan (1990): "El género: una categoría útil para el analista histórico", en AMELANG, James \& NASH, Mary (eds.): Historia y género. Las mujeres en la Europa Moderna y Contemporánea, Valencia, Edicions Alfons el Magnànim, pp: 23-56.

VALCARCEL, Amelia (2002): Ética para un mundo global. Una apuesta por el humanismo frente al fanatismo, Madrid, Temas de Hoy.

WOLLSTONECRAFT, Mary (1998): Vindicación de los derechos de la mujer, Madrid, Debate.

WOOD, Wendy \& EAGLY, Alice H. (2002). "A Cross cultural analysis of the behaviour of women and men: implications for the origins of sex differences", Psychological bulletin, vol 128(5): 699-727.

XIFRA TRIADÚ, Xordi (2010): Relaciones Públicas, empresa y sociedad: una aproximación ética, Barcelona, UOC.

[Recibido: 4 de noviembre de 2014. Aceptado: 8 de febrero de 2016] 